\title{
Horizontale Integration: Wo liegen die Vorteile, wo die Knacknüsse?
}

\section{Michael Zaugg}

Prof. Dr. med., MBA, University of Alberta, Kanada
Die Schweiz (8 Mio. Einwohner, BIP 660 Mia., Gesundheitskosten 11,5\% des BIP) hat mit 300 Spitälern im Jahre 2011 noch immer eine sehr hohe Spitaldichte (Abbildung 1). Auch wenn die Anzahl kleiner Spitäler $(<100$ Betten) in der Grundversorgung von 166 auf $90(-46 \%)$ über die letzten 10 Jahre markant abgenommen hat [1], scheint eine weitere Reduktion bei einer mittleren Bettenbelegung von 92\% und einer schon relativ kurzen (mittleren) Spitalaufenthaltsdauer von 6,6 Tagen (2011, in der Grundversorgung) eher unrealistisch. Erfahrungen aus anderen Gesundheitssystemen zeigen jedoch, dass bedeutende Kosteneinsparungen durch Konsolidierung im Spitalmarkt mittels Spitalfusionen möglich sind. Dies geht im Regelfall mit einer Schliessung und/oder Umnutzung von Überkapazitäten einher. Weniger und grössere «Spitaleinheiten» ersetzen so mehrere kleinere.

Der Druck aus der Politik [2], die klar weniger, aber profitablere Spitäler mit ausgebautem qualitativ hochstehendem Leistungsangebot will, die zunehmende Konkurrenz zwischen den Spitälern aufgrund der neuen prospektiven DRG-basierten Spitalfinanzierung, ein absehbarer Ärztemangel und der Ruf nach Mindestgrössen begünstigen diese Entwicklung. Im Moment kommt es in der Schweizer Spitallandschaft zu einem massiven Ausbau der Spitalnetzwerke.

Die ökonomischen Konsequenzen von Netzwerken versus Spitalfusionen sind aber sehr verschieden. Horizontale Integration im Spitalwesen betrifft immer mehr auch die Ärzteschaft in und um die Spitäler, welche diese mitzutragen und oft auch zu implementieren hat. Der folgende Artikel diskutiert die ökonomischen Konsequenzen der verschiedenen Formen der horizontalen Integration, die Faktoren, die $\mathrm{zu}$ einer erfolgreichen horizontalen Integration beitragen, und stellt diese in den schweizerischen Kontext.

\section{Ökonomische Vorteile durch horizontale Integration}

Horizontale Integration im Spitalwesen ist das $\mathrm{Zu}$ sammenlegen und/oder die Netzwerkbildung von Spitälern. Dabei kann es sich um eine rechtlich relativ lose Zusammenarbeit («Multihospital Affiliation») oder um eine komplette rechtliche Fusion der individuellen Partner handeln («Merger») (Tabelle 1).

\section{L'intégration horizontale:} ses avantages et ses casse-tête

La Suisse a une densité hospitalière qui reste très élevée avec 300 hôpitaux en 2011 (fig. 1). Même si le nombre de petits hôpitaux ( $<100$ lits) a fortement diminué dans les soins de base, passant de 166 à 90 ( $-46 \%)$ au cours des 10 dernières années [1], une réduction supplémentaire semble plutôt irréaliste si l'on considère que le taux moyen d'occupation des lits est de $\mathbf{9 2} \%$ et que la durée (moyenne) d'hospitalisation est déjà relativement brève $(6,6$ jours dans les soins de base en 2011). L'expérience recueillie dans d'autres systèmes de santé montre toutefois qu'une consolidation du marché hospitalier obtenue par des fusions d'hôpitaux permet de réaliser des économies substantielles. Une telle mesure implique en règle générale une fermeture d'établissement et/ou une réaffectation des surcapacités. Plusieurs petites «unités hospitalières» en viennent ainsi à être remplacées par de plus grandes. Cette évolution est favorisée par la pression des milieux politiques [2]. Nous assistons actuellement à un développement massif des réseaux dans le paysage hospitalier suisse. Or, les conséquences économiques des réseaux sont très différentes de celles des fusions d'hôpitaux. L'intégration horizontale dans le secteur hospitalier implique aussi toujours davantage les médecins employés ou accrédités par les hôpitaux, qui doivent cautionner cette intégration et souvent participer à sa mise en œuvre. L'article discute des conséquences économiques des différentes formes d'intégration horizontale et des facteurs qui contribuent au succès d'une intégration horizontale réussie, et place ceux-ci dans le contexte suisse. 


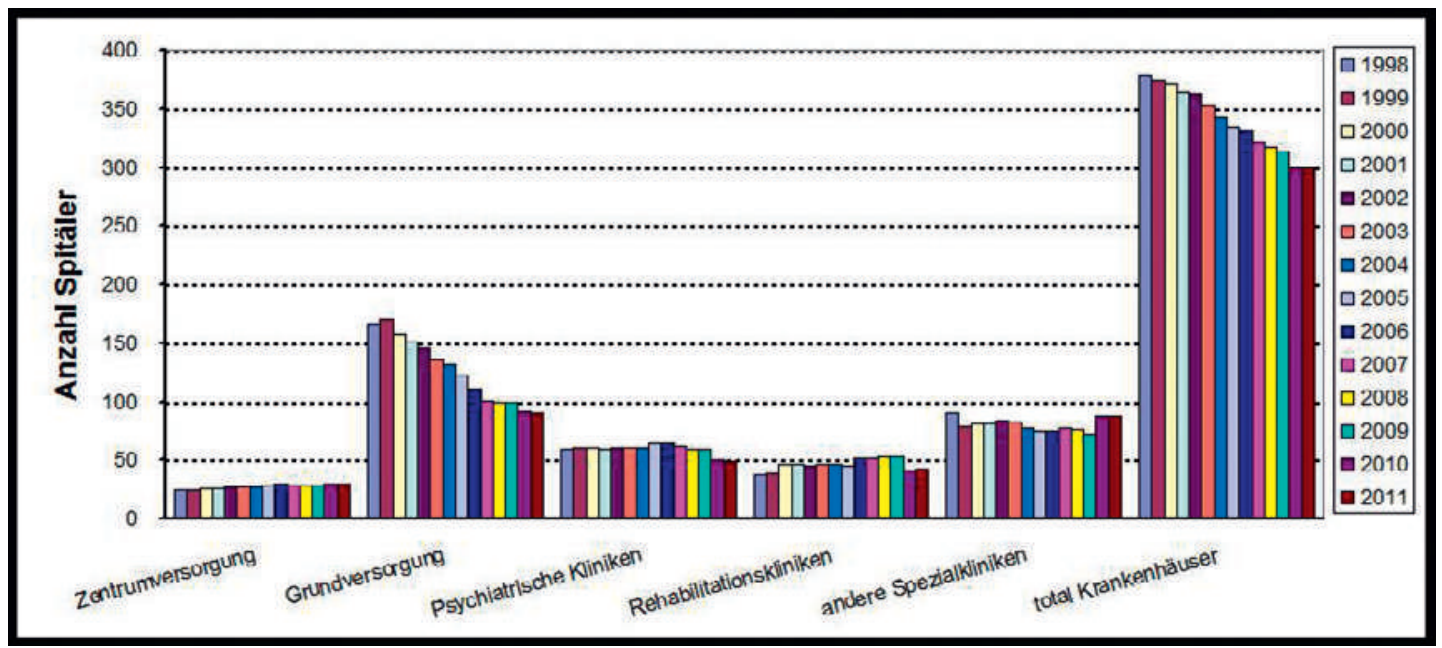

Abbildung 1

Anzahl Spitäler (1998-2011) in der Schweiz, aufgeteilt nach Grundversorgung, Zentrumversorgung, Psychiatrie, Rehabilitation und anderen Spezialkliniken (Bundesamt für Statistik).

Nach der klassischen Lehre der Ökonomie führt die horizontale Integration zu Skalen- und PortfolioEffekten. Fixe Kosten (Infrastruktur, Personal, Administration, Finanzen, Einkauf, Technologie, Marketing) werden dabei auf mehrere oder verschiedene Dienstleistungen aufgeteilt. Schätzungen gehen von Kosteneinsparungen von 5-20\% aus [3]. Horizontale Integration führt zu I) einer Verbesserung der finanziellen Voraussagbarkeit mit höherer Stabilität, II) einem erweiterten, attraktiveren Serviceangebot und III) einer gesteigerten operativen Effizienz durch Beseitigung von Doppelspurigkeiten und Überkapazitäten. Durch Fusion nimmt in grösseren Betrieben das sog. «peak load staffing» (erforderliche Anzahl Festangestellter, um 95\% des Patientenanfalls in Spitzenzeiten zu behandeln) wegen geringerer Fluktuation im Bedarf ab, was die Fixkosten verringert.
Weitere wichtige Vorteile sind der grössere Marktanteil und die dadurch verbesserte Verhandlungsposition (Marktmacht) beim Geschäften mit Zulieferern und Versicherern. Empirische Studien und Fallstudien aus den USA zeigen, dass die grössere Marktmacht und nicht etwa ein allfälliges hausgemachtes Defizit die treibende Kraft zur horizontalen Integration ist. Offenbar steht der Wunsch, die eigene operative Effizienz zu verbessern, weniger im Vordergrund. Dies mag erklären, weshalb gerade grössere und nicht etwa nur kleinere finanziell schwächere Spitäler sich «horizontal besser vernetzen» wollen.

Horizontale Integration verringert zudem das finanzielle Risiko, verbessert die Möglichkeiten der Quersubventionierung bei unvorhergesehenen Verlusten und erleichtert das Auftreiben von Kapital für

Tabelle 1

Ökonomische Konsequenzen verschiedener Formen von horizontaler Integration im Spitalwesen.

\begin{tabular}{lll} 
& $\begin{array}{l}\text { Spitalfusion } \\
\text { (Hospital Merger) }\end{array}$ & $\begin{array}{l}\text { Spitalnetzwerk } \\
\text { (Multihospital Affiliation) }\end{array}$ \\
\hline Rechtliche Struktur & $\begin{array}{l}\text { oft } \\
\text { komplette rechtliche Fusion }\end{array}$ & $\begin{array}{l}\text { variabel: } \\
\text { von rechtlich bindenden } \\
\text { Verträgen bis zu rechtlich wenig } \\
\text { bindenden Abmachungen }\end{array}$ \\
\hline Kostenreduktion & + & $(+)$ \\
\hline Operative Effizienz & + & $(+)$ \\
\hline Marktmacht (Preise/Erträge) & + & + \\
\hline $\begin{array}{l}\text { Abbau von Überkapazität } \\
\text { (Teilschliessungen/Umnutzungen) }\end{array}$ & + & - \\
\hline Serviceangebot (Portfolio) & & + \\
\hline $\begin{array}{l}\text { Servicequalität } \\
\text { Hang zu mehr Quersubventionierung }\end{array}$ & + & $(=) ?$ \\
\hline $\begin{array}{l}\text { Hang zu erweiterter Indikationsstellung und mehr } \\
\text { Dienstleistungen (Quantität) }\end{array}$ & - & + \\
\hline
\end{tabular}


Neuinvestitionen. Andererseits kann sie aber auch zu Ineffizienz führen, wenn der Betrieb zu gross wird. In diesem Fall spielen Probleme in der Kommunikation und der Motivation der Mitarbeiter eine wichtige Rolle. Als Faustregel gilt, dass Spitaleinheiten mit 10000 Hospitalisationen pro Jahr bezüglich Kosten optimiert werden können. Komplett unklar ist aber, bis zu welcher Grösse und bis zu welchem Grad der Komplexität Spitalnetzwerke operative und finanzielle Vorteile mit sich bringen.

\section{Wie wird der Erfolg der horizontalen Integration objektiviert?}

Bis heute fehlen Analysen, die den Prozess des Organisationswandels bei horizontaler Intergration im Spitalwesen mit dem Schlussresultat (Outcome) verbinden. Die meisten Studien messen den Erfolg der horizontalen Integration auf den folgenden drei Ebenen [3], die aber in ihrer Aussage limitiert sind:

1. Kosten: Ein beliebter Indikator sind die Kosten in den ersten drei Jahren nach erfolgter horizontaler Integration, was aber eigentlich nur einem Kurzzeitverlauf entspricht. Die Resultate in der Literatur zeigen, dass Spitalfusionen in Gegensatz zu Netzwerken immer mit Kosteneinsparungen einhergehen. Die Kosteneinsparungen sind aber schnell ausgeschöpft, meist limitiert auf kleinere bis mittelgrosse Spitäler und hauptsächlich auf eine administrative Optimierung zurückzuführen.

2. Erträge und Profitabilität: Die meisten Studien berichten von höheren Erträgen und meistens auch gesteigerter Profitabilität, und zwar unabhängig davon, ob es sich um eine Spitalfusion oder um ein Spitalnetzwerk handelt. Verantwortlich hierfür sind der höhere Marktanteil und die höhere Marktmacht.

3. Servicequalität: Dieser für Patienten und Ärzteschaft wichtige Outcome zeigt in der Literatur keine wesentlichen Veränderungen, und die bisherigen Studien haben keine Qualitätsverbesserung gefunden. Vereinzelt wird eher über eine höhere Wiedereintritts- oder Frühentlassungsrate berichtet. Gemäss Literatur beeinflussen Spitalfusionen die Hospitalisationsmortalität nicht. Unbestritten ist der Vorteil einer höheren Fallzahl in der hochspezialisierten Medizin.

Wollen Sie diesen Artike kommentieren? Nutzen Sie dafür die Kommentarfunktion in der OnlineVersion oder sehen Sie nach, was Ihre Kolleginnen und Kollegen bereits geschrieben haben: www.saez.ch/ aktuelle-ausgabe/ interaktive-beitraege/

\section{«Faktoren zum Erfolg»}

Eine horizontale Integration folgt idealerweise einem strukturierten zeitlichen Ablauf [3]. Zuerst erfolgt Integration auf der Ebene der Administration, dann der dienstleistungsunterstützenden Aktivitäten. Schliesslich können die klinischen Dienstleistungen (zuletzt die Massendienstleistungen) integriert werden. Bei Fusionen wird zuerst in der Administration und beim Senior Management Überkapazität abgebaut und erst zuletzt im Kerngeschäft bei der Pflege und/oder der Ärzteschaft.
Dabei ist zu bedenken, dass Abbau von medizinischen Dienstleistungen und/oder Standortverschiebungen oft mit grossem internem Widerstand einhergehen. Mehr Hierarchie im administrativen Bereich macht Veränderungen hier einfacher. Bei Umstrukturierungen im Bereiche der medizinischen Leistungen ist Leadership mit medizinischer und ManagementKompetenz von zentraler Bedeutung (Führen von Experten). Ein ärztlicher Direktor (chief medical officer, CMO), der im Sinne der Geschäftsleitung und der Strategie des Verwaltungsrates geschickt agiert, kann hier viele Konflikte vermeiden oder anstehende Probleme effizient lösen.

Um eine erfolgreiche Integration von medizinischen Dienstleistungen zu erreichen, braucht es zuerst eine Integration der verschiedenen «lokalen Kulturen», was oft nur langsam vonstatten geht. Die Erfahrung zeigt, dass es einen anhaltenden ökonomischen Druck von aussen braucht, wenn schwierige Entscheidungen (Abbau von Doppelspurigkeiten) anstehen. Zudem vereinfacht das Einsetzen einer Task Force aus Entscheidungsträgern aller zu «integrierenden» Spitäler/Abteilungen die Aufgabe entscheidend. Die Task Force soll dem gemeinsamen Ziel der Effizienz kompromisslos verpflichtet sein und gemeinsame Werte und Visionen für die Zukunft erarbeiten.

Schliesslich braucht es eine unermüdliche transparente Kommunikation, die alle Beteiligten miteinbezieht. Bisherige Studien zeigen, dass die Fusion gleich grosser Spitäler die operative Effizienz am meisten steigert. Dieser Mehrwert scheint weniger ausgeprägt zu sein bei Netzwerkbildung, doch Netzwerke erlauben profitablen Spitälern, ihre Gewinne selbst zu behalten. Eine gute Voraussetzung für das Gelingen der Integration ist die geographische Nähe der zu integrierenden Einheiten und das Fehlen vorbestehender Konflikte. Das Einstellen von Akutserviceleistungen führt häufig zu grossem lokalem politischem Widerstand, den man nur durch Einbezug der Betroffenen überwinden kann.

Leider zeigen viele Studien über Spitalfusionen und Netzwerkbildungen, dass gerade die Integration von medizinischen Leistungen ungenügend erfolgt. Engagement, strategische Planung, Kommunikation, Benchmarking mit integrativen und koordinierenden Aktivitäten sind von zentraler Bedeutung, da sie die Betroffen einbeziehen und so die Akzeptanz zur Organisationsveränderung erhöhen. Hilfreich sind dabei auch attraktive Anreizsysteme für die Mitarbeiter.

\section{Erfahrungen aus zwei EU-Kleinstaaten}

1. Das Dänische Modell (5,6 Mio. Einwohner, BIP 335 Mia., Gesundheitskosten 11,4\% des BIP): Dänemark hat ein integriertes Gesundheitswesen, das in 5 Regionen eingeteilt ist und vom Staat finanziert wird (Typ Beveridge) [4]. Diese Regionen haben die operative and strategische Verantwor- 
tung für die Spitäler. Die Zahl der Akutspitäler sank von 1980 bis 2007 von 117 auf 35, auch begünstigt durch die Einführung eines DRG-basierten Spitalfinanzierungssystems im Jahre 2002. Nach 2007 hat eine staatliche Initiative, breitabgestützt durch das sog. «National Board of Health», die Anzahl der Akutspitäler weiter von 35 auf 20 reduziert. Die massive Restrukturierung mit ca. 5 Mia. $€$ Aufwand beinhaltete nicht nur das Schliessen oder Modernisieren von bestehenden Spitalanlagen, sondern auch das Bauen von neuer, modernerer Infrastruktur ("greenfield investments»). Durch diese Konsolidierung konnte das dänische Gesundheitswesen langfristige Kosteneinsparungen von bis zu 20\% erreichen.

2. Das Österreichische Modell (8,4 Mio. Einwohner, BIP 420 Mia., Gesundheitskosten 11\% des BIP): Hier hat das Österreichische Bundesinstitut für Gesundheitswesen einen Masterplan erarbeitet, der von den 9 Bundesländern implementiert wurde (32 Gesundheitsregionen/4 Gesundheitszonen) [5]. Weil viele der Gemeindespitäler defizitär waren und die Gemeinden für die Spitäler Defizitgarantien abgeben mussten, wurde von den Gemeinden die Hoheit über die Spitäler gerne an die Bundesländer abgegeben. Dabei spielte die DRG-basierte Spitalfinanzierung eine wichtige Rolle, weil sie zu einer Reduktion der mittleren Hospitalisationszeit, zu einer Bettenunterbelegung und schliesslich zum Defizit der Spitäler führte. Betriebe wurden neu zusammengefasst unter Privatrecht und einem einzigen Management unterstellt. Einige Spitäler wurden auch geschlossen oder umgenutzt. Diese Entwicklung führte zwischen 1997 und 2003 zu einer 8\%-Abnahme der Bettenkapazität.

\section{Überlegungen zur horizontalen Integration in der Schweiz}

Da die Spitäler den Löwenanteil der Gesundheitskosten verursachen, fordert die Politik eine Reduktion der Überkapazität. In den meisten Spitalmärkten wie bei unserem Nachbarn Deutschland scheinen DRG und kürzere Hospitalisationszeit die Infrastrukturüberkapazität abzubauen. Studien aus dem europäischen Umfeld aus Gesundheitssystemen mit vergleichbarer Grösse (Dänemark, Österreich) zeigen, dass die horizontale Integration im Rahmen grosser Restrukturierungen erfolgreich eingesetzt wurde. Dabei war aber ein aktives Eingreifen des Staates in den freien Markt mit dem Erstellen eines Masterplans zur Spitallandschaft vonnöten.

Eine Spitalplanung findet zwar auch in der Schweiz kontinuierlich auf kantonaler Ebene statt, ist aber im Ansatz viel föderalistischer. Nach einer Phase der Konsolidierung in der schweizerischen Spitallandschaft ist eine weitere Abnahme der Anzahl Spitäler in der Schweiz eher nicht zu erwarten. Im Moment schreitet aber die Netzwerkbildung unter Schweizer Spitälern weiter voran. Die ökonomischen Konsequenzen einer Netzwerkbildung versus
Spitalfusionen sind aber grundsätzlich verschieden. Es scheint, dass der DRG-bedingte vermehrte «Wettbewerb» und die Netzwerkbildung unter Spitälern eher zu mehr medizinischen Leistungen (erweiterte Indikationen) [6] und einem Hang zur Quersubventionierung (wegen zu knapper Kostendeckung insbesondere bei allgemeinversicherten Patienten) führen [7]. Sollten sich diese Tendenzen weiter bewahrheiten, ist dies bedenklich und sicher nicht im Interesse der Patienten. Leider liegen keine aktuellen detaillierten Studien über Formen und Aktivitäten der horizontalen Integration im schweizerischen Spitalwesen bis dato vor. Die Entwicklung der horizontalen Integration sollte aber gerade im Zusammenhang mit der neuen Spitalfinanzierung monitorisiert werden. Der Markt alleine kann die Anzahl der Spitäler wohl kaum weiter reduzieren, da zu viele Partikularinteressen im Spiel sind. So sind Spitäler oft wichtige lokale Arbeitgeber und Wirtschaftsmotoren. Auf der anderen Seite zeigt die Erfahrung aus anderen Gesundheitssystemen aber, dass wirkliche horizontale Integration von medizinischen Leistungen mit Optimierung der Ressourcen, nur mit dem Zusammenlegen von Infrastrukturen (eventuell mit Teilschliessungen/Umnutzungen) und einem gewissen Abbau von Überkapazitäten im medizinischen Leistungsangebot zu erreichen ist. Die Politik und der Geldbeutel des Einzelnen werden schliesslich über die Richtung in der Gesundheitspolitik und die Anzahl der Spitäler in der Schweiz entscheiden.

\section{Referenzen}

1. Bundesamt für Statistik http://www.bfs.admin.ch/bfs/portal/de/index/ themen/14/03/01.html (1/5/2013).

2. Gesundheitsdirektor Thomas Heinigers (FDP) Vorschlag, das Unispital mit den Zürcher Stadtspitälern Triemli und Waid zu fusionieren, erntet grossen Applaus. Einzelne Politiker würden gar noch weiter gehen. http://www.tagesanzeiger.ch/zuerich/region/ Stadtraetin-Nielsen-findet-Heinigers-Spitalfusionsplaene-pruefenswert/story/31642912 (30/10/2013).

3. Bazzoli GJ, Dynan L, Burns LR. Two decades of organizational change in health care: what have we learned? Medical Care Research and Review. 2004;61:247-331

4. Kristensen T, Olson KR, Kilsmark J, Lauridsen JT, Pedersen KM. Economies of scale and scope in the Danish hospital sector prior to radical restructuring plans. Health Policy. 2012;106:120-126.

5. Fidler AH, Haslinger RR, Hofmarcher MM, Jesse M Palu T. Incorporation of public hospitals: A «Silver Bullet» against overcapacity, managerial bottlenecks and resource constraints? Case studies from Austria and Estonia. Health Policy. 2007;81:328-338.

6. SonntagsZeitung Ausgabe 6. Januar 2013. Das kranke System. http://www.sonntagszeitung.ch/fokus/ artikel-detailseite/?newsid=239667 (1/5/2013).

7. Handelszeitung Ausgabe 14. März 2013. Fallpauschalen bald vor Gericht. http://www.handelszeitung.ch/ ausgabe/handelszeitung-1311 (1/5/2013).

(Weitere Referenzen sind erhältlich auf Anfrage.) 